\title{
Armazenamento Oportunista em Redes de Dados Nomeados Sem Fio como Suporte à Mobilidade de Produtores
}

\author{
Francisco R. C. Araújo ${ }^{1}$, Antonio M. de Sousa ${ }^{1}$, Leobino N. Sampaio ${ }^{1}$ \\ ${ }^{1}$ Programa de Pós-Graduação em Ciência da Computação (PGCOMP) \\ Departamento de Ciência da Computação - Universidade Federal da Bahia (UFBA) \\ Salvador - BA - Brasil \\ \{franciscorca, antonio.mateus, leobino\}@ufba.br
}

\begin{abstract}
The Information-Centric Networking were proposed in order to meet the new demands of Internet traffic, among its various approaches Named Data Networking (NDN) are the most investigated and, while supporting the mobility of consumers, the mobility of the producers still represents a challenge given the cost of upgrading the routes on the nodes and, even more critical, the producer's absence periods. In this context, this work proposes to minimize the effects of the mobility of producers in NDN, through an interest forwarding strategy and a cache replacement policy for the nodes of the wireless network. The solution was implemented in the ndnSIM simulator, and the results show gains of up to $35.32 \%$ in the interest satisfaction rate with time reduction of $56.23 \%$ compared to the default NDN.
\end{abstract}

Resumo. As Redes Centradas na Informação foram propostas na perspectiva de atender as novas demandas de tráfego da Internet, dentre suas várias abordagens as Redes de Dados Nomeados (NDN) são as mais investigadas e, embora suporte a mobilidade dos consumidores, a mobilidade dos produtores ainda representa um desafio, dado o custo de atualização das rotas nos nós e, ainda mais crítico, os períodos de ausência do produtor. Nesse contexto, este trabalho propõe minimizar os efeitos da mobilidade de produtores em NDN, através de uma estratégia de encaminhamento de interesses e uma política de substituição de cache para os nós da rede sem fio. A solução foi implementada no simulador ndnSIM, e os resultados mostram ganhos de até 35,32\% na taxa de satisfação de interesses com redução de tempo de 56,23\% comparada à NDN padrão.

\section{Introdução}

Dentre as Redes Centradas na Informação se destacam as Redes de Dados Nomeados (do inglês, Named Data Networking - NDN), as quais foram propostas com o objetivo de atender as demandas de tráfego atuais e futuras da Internet (e.g., os 49 exabytes mensais previstos até 2021 [Cisco 2017]), através de uma arquitetura baseada no modelo de comunicação centrado no conteúdo. NDN possui consumidores que requisitam pacotes de dados aos produtores de conteúdo, por meio do envio de pacotes de interesse. Tais pacotes atuam em conjunto com três estruturas básicas: PIT (do inglês, Pending Interest Table), FIB (do inglês, Forwarding Information Base) e CS (do inglês, Content Store), que respectivamente mantêm os interesses ainda não satisfeitos, as rotas para as fontes de dados e o armazenamento de dados na cache dos dispositivos [Zhang et al. 2014]. 
A mobilidade dos consumidores é suportada intrinsecamente pela NDN, estes podem, simplesmente, emitir interesses pendentes a partir de seus novos locais, uma vez que os conteúdos podem ser encontrados na cache em diferentes partes da rede. Por outro lado, a mobilidade dos produtores é desafiadora. Em tais redes, os produtores precisam anunciar seus novos locais após o handoff, o que causa a poluição das entradas na FIB dos nós intermediários [Huynh et al. 2017]. [Lehmann et al. 2016] caracterizam a mobilidade dos produtores em dois períodos: indisponibilidade e reconexão. O primeiro consiste na falta de conectividade de rede do produtor, o segundo refere-se ao processo de restaurar a conectividade do mesmo. Embora a arquitetura da NDN suporte a fase de reconexão, a indisponibilidade não é tratada e, portanto, carece de maiores investigações, sobretudo por conta da crescente adoção e predomínio das redes móveis.

A carência de soluções para tratar a indisponibilidade de conteúdos em cenários de redes móveis, tem motivado alguns trabalhos recentes [Lehmann et al. 2016, Farahat and Hassanein 2016, Ge et al. 2016]. Os mesmos propõem resolver o problema da mobilidade de produtores em NDN, contudo, a partir da alteração da característica reativa da rede (i.e., receber interesse para encaminhar dados) ou em desacordo com o princípio de centralidade no conteúdo. Além disso, tais propostas não consideram o armazenamento de dados nos próprios nós da WLAN (do inglês, Wireless Local Area Network). Outras iniciativas têm investigado a capacidade computacional e cooperação dos nós na borda da rede [Panwar et al. 2017, Rehman and Kim 2017, Hahm et al. 2017], entretanto, para propósitos diferentes ao desta pesquisa.

Diante dessas questões, este trabalho apresenta uma proposta que busca minimizar os efeitos da mobilidade de produtores, através do armazenamento oportunista de dados na WLAN. Diferente de algumas abordagens existentes, a proposta é centrada no conteúdo e segue os princípios do paradigma da NDN. Assim, os dispositivos presentes na rede sem fio armazenam os dados do produtor sem esforço, de forma a explorar a característica broadcast da rede (i.e., oportunista). Dessa forma, sempre que um produtor responde a uma requisição, este seleciona um dispositivo no domínio para armazenar o dado. Portanto, obtém-se uma maior diversidade de conteúdos na rede, o que aumenta as chances de resposta aos interesses que chegam em períodos de indisponibilidade do produtor. A proposta é totalmente distribuída e, embora seja avaliada em um ambiente de WLAN, pode ser aplicada a outros cenários de redes sem fio (e.g., 5G, Mesh, Ad Hoc).

Em resumo, as principais contribuições deste trabalho são as seguintes: i) uma nova estratégia de encaminhamento de interesses que minimiza os efeitos do broadcast storm; ii) uma nova política de substituição de cache que prioriza os dados dos produtores propensos a efetuarem handoff. Além disso, a proposta foi avaliada com base em um estudo experimental. O ambiente de experimentação foi desenvolvido no simulador ndnSIM [Mastorakis et al. 2017], onde foram reproduzidas WLANs infraestruturadas formadas por consumidores e produtores móveis. Neste ambiente, foram consideradas as métricas taxa de satisfação de interesses e tempo de obtenção de dados a partir da variação do número de produtores em handoff e da velocidade dos mesmos. Os resultados dos experimentos mostraram que a solução proposta obtém 35,32\% em ganho na entrega de dados e com uma redução de tempo de 56,23\% comparada à NDN padrão.

O restante deste trabalho está organizado da seguinte forma: a Seção 2 apresenta os trabalhos relacionados e a Seção 3 descreve a solução proposta. Na Seção 4 os expe- 
rimentos e resultados são apresentados e, por fim, a Seção 5 apresenta as considerações finais e discute os trabalhos futuros.

\section{Trabalhos Relacionados}

O problema da mobilidade de produtores em NDN tem sido investigado por alguns trabalhos recentes [Zhang et al. 2016]. Em [Lehmann et al. 2016] os autores propuseram um método de replicação proativa de dados dos produtores que considera os nós da vizinhança para armazena-los. No entanto, devido a caraterística reativa da arquitetura da NDN, foi necessária a criação de um novo tipo de pacote (chamado Di ca). Este por sua vez, tem a função de informar aos nós que solicitem dados a um determinado produtor. Isso implica que para cada pacote de dados replicado são necessários dois pacotes extras, um interesse e um Dica. Dessa forma, há uma sobrecarga na rede pelo tráfego desses pacotes e, também, uma sobrecarga no produtor que precisa replicar seus dados periodicamente.

Diferente do trabalho apresentado, a solução proposta explora a capacidade da rede sem fio, de modo a tirar proveito da comunicação broadcast, isso dispensa pacotes extras para obter os dados do produtor. Além disso, evita-se que o produtor se sobrecarregue para replicar dados, ou seja, os dados que são respondidos pelos produtores, na solução proposta, são armazenados na cache dos nós da WLAN de maneira oportunística. Assim, evita-se pacotes extras na rede e sobrecarga no produtor. Isso implica que os dados que são replicados são aqueles que foram solicitados ao menos uma vez. O que é justificável já que dados não requisitados não têm popularidade, logo não são replicados.

No trabalho de [Farahat and Hassanein 2016] é apresentada OpCacheMob, uma solução proativa de replicação de dados, baseada na predição de localização dos produtores móveis e dos interesses destinados aos mesmos. Os autores propuseram uma divisão na cache dos nós, com uma parte destinada aos conteúdos oriundos de produtores móveis, na tentativa de priorizar os dados dos mesmos. A OpCacheMob trata o período de indisponibilidade do produtor e sua característica proativa, como no trabalho anterior, causa as mesmas sobrecargas à rede e aos produtores. Além disso, diferente da solução proposta, ignoram o armazenamento nos nós da WLAN.

No trabalho [Ge et al. 2016], os autores integraram o paradigma das Redes Definas por Software (do inglês, Software Defined Networking - SDN) à NDN. O trabalho trata a fase de reconexão do produtor, por meio de atualizações das entradas da FIB dos nós, utilizando a visão global do controlador SDN. Porém, o fato de envolver a figura do controlador confronta o princípio da NDN. Em contraste, a solução proposta se concentra na fase de indisponibilidade do produtor pelo fato desta não ter suporte da arquitetura da NDN, já na fase de reconexão assume-se o procedimento da NDN padrão.

Por fim, trabalhos recentes têm investigado NDN em cenários de MANETs [Rehman and Kim 2017]; explorado ICN na borda da rede [Panwar et al. 2017]; e em dispositivos de capacidades computacionais variadas na borda da rede, IoT [Hahm et al. 2017]. Esses trabalhos consideram os recursos e a cooperação dos dispositivos móveis o que mostra que a solução proposta tem potencial e relevância.

\section{Solução Proposta}

Este trabalho apresenta uma solução que visa minimizar os impactos causados na rede pela mobilidade de produtores em NDN, através do uso oportunista da cache dos nós 
presentes na WLAN. O objetivo é proporcionar que os produtores possam replicar seus dados, de forma a manter a maior diversidade de conteúdos, entre os nós e que estes possam armazenar os dados oportunisticamente, para que os interesses futuros possam ser satisfeitos mesmo durante o período de indisponibilidade do produtor.

A solução se baseia no princípio básico de armazenar os dados que trafegam na rede, de modo a evitar as replicações em todos os nós do domínio, a fim de alcançar uma maior diversidade de conteúdos. Os dados são armazenados sem esforço e oportunisticamente, pois os nós se aproveitam da característica broadcast da comunicação das redes sem fio. Por outro lado, essa característica pode causar a inundação de pacotes na rede e a solução bloqueia essas retransmissões. Obtém-se, portanto, o livre broadcast quando o pacote é do tipo dado e bloqueio quando é do tipo interesse. [Zhang et al. 2016] destacam que na NDN os dados podem ser facilmente retirados dos produtores originais, uma vez que são nomeados, além disso a proteção é aplicada aos mesmos e não ao meio de acesso.

Para desenvolver a solução foram necessárias pequenas modificações na arquitetura da NDN, como ilustrado na Figura 1. A arquitetura da NDN padrão está representada na Figura 1 - I. Nota-se que na camada de aplicação (i.e., topo da pilha) foi adicionado um novo tipo de pacote para o envio de sondas (probes) para descoberta de nós no domínio (Figura 1 - II (a)) e um acréscimo de dois campos, idNodeBackup e priorityData, no pacote de dados para manter, respectivamente, o identificador do nó que deve armazenar a réplica do dado e a prioridade do mesmo (Figura 1 - II (b)). Com isso, evita-se criar um novo tipo de pacote específico, destinado apenas, para desempenhar o controle da solução, o que resultaria na geração de mais tráfego na rede. Outros acréscimos ocorreram na camada de estratégia, através de uma nova estratégia de encaminhamento, uma nova política de substituição de cache e uma simples estrutura (Tabel $a_{\text {vizinhos }}$ ) para manter em cada nó a lista dos demais presentes na WLAN (Figura 1 - II (c)).

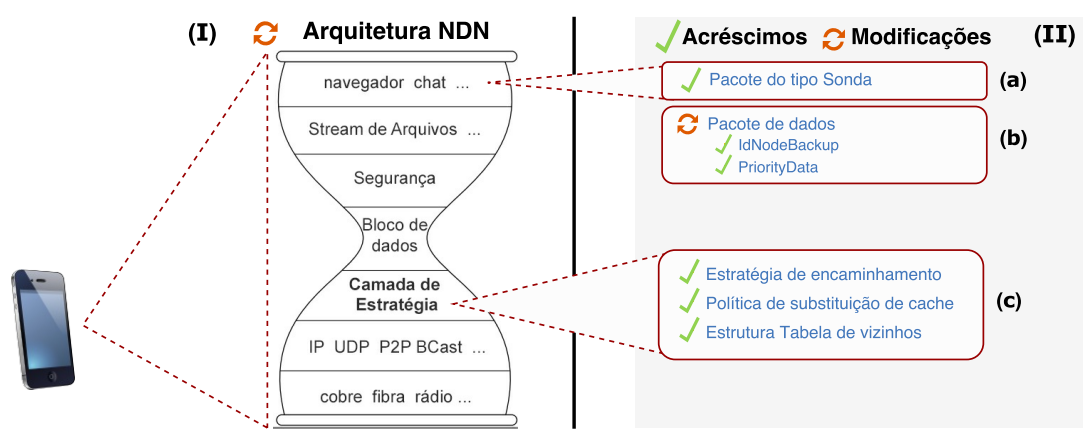

Figura 1. Modificações e acréscimos na arquitetura da NDN.

A modificação mais significativa na arquitetura da NDN foi o acréscimo do pacote de sondas e da estrutura Tabel $a_{\text {vizinhos }}$. No entanto, de acordo com os resultados obtidos, tais mudanças não representam maiores prejuízos à rede. Os detalhes das funcionalidades da solução são apresentados nas próximas subseções.

\subsection{Envio Periódico de Sondas}

As sondas se baseiam em um identificador (id), em cada nó da WLAN, com o intuito principal de possibilitar a descoberta de vizinhos de até um salto. Isso não contraria os princípios da NDN, pois o id pode ser o próprio endereço MAC dos nós. O prefixo não 
foi utilizado como identificador, pois os nós recém-ingressantes ou nós não produtores não teriam prefixo para anunciar e, portanto, não seriam conhecidos pelos seus vizinhos.

Uma vez identificados, os nós precisam anunciar sua presença aos demais nós da rede local. Para possibilitar esse anúncio, a solução proposta incorpora um novo tipo de pacote, chamado sonda, que foi implementado na camada de aplicação como uma variante do pacote de interesse. No entanto, em razão da sua função básica de apenas informar a presença do nó na rede, sua carga útil é vazia, o que o torna bastante leve. A informação que esse pacote transporta é inserida no campo destinado ao nome do mesmo, da seguinte forma: / id, o caractere / é utilizado pela estratégia de encaminhamento do nó para enviar o pacote na rede e o id representa o próprio identificador do nó.

Periodicamente os nós da WLAN enviam pacotes sondas, que são recebidos por cada nó no domínio local via broadcast. Ao receber um pacote desse tipo o nó extrai a informação id do nome do pacote e a insere ou atualiza a entrada em sua estrutura

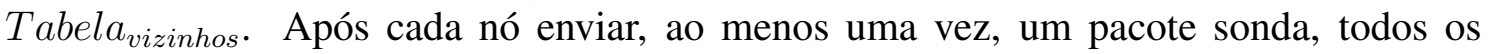
outros conseguem obter a informação id dos demais, presentes na rede local, em sua tabela Tabela $a_{\text {vizinhos. }}$. Ou seja, as sondas preenchem e mantêm a estrutura Tabelavizinhos atualizada em cada nó.

\subsection{Estratégia de Encaminhamento de Interesses}

$\mathrm{Na}$ arquitetura da NDN sempre que um determinado nó recebe um interesse, sua cache é verificada em busca do dado correspondente, se este nó não possuir o respectivo dado, o mesmo deve encaminhar o interesse na rede [Zhang et al. 2014]. Para encaminhar interesses é utilizada uma estratégia de encaminhamento. A NDN padrão possui algumas estratégias como, por exemplo, best-route e multicast [Mastorakis et al. 2017].

Na solução proposta foi desenvolvida uma nova estratégia de encaminhamento de interesses que bloqueia o reencaminhamento desses pacotes. A fim de minimizar os efeitos do broadcast storm. Interesses podem ser encaminhados em até um salto dentro do domínio, pois em um ambiente de rede local, o envio de pacotes com um salto, significa que os nós presentes no domínio são alcançados com este pacote. Logo, os reencaminhamentos dentro do domínio, representam uma redundância desnecessária que prejudica o desempenho da rede, através da inundação de pacotes. A lógica do funcionamento da estratégia de encaminhamento desenvolvida está representada na Figura 2. Nota-se que há o encaminhamento, na rede, de pacotes de interesse e de sondas que vêm da aplicação local. Além do envio de pacotes destinados à aplicação local para a camada correspondente. Porém, os pacotes que não atendem as condições mencionadas são descartados. Além disso, as sondas que chegam da rede são passadas à estrutura Tabel $a_{\text {vizinhos }}$, para a inserção ou atualização dos identificadores dos nós vizinhos, em seguida são descartadas.

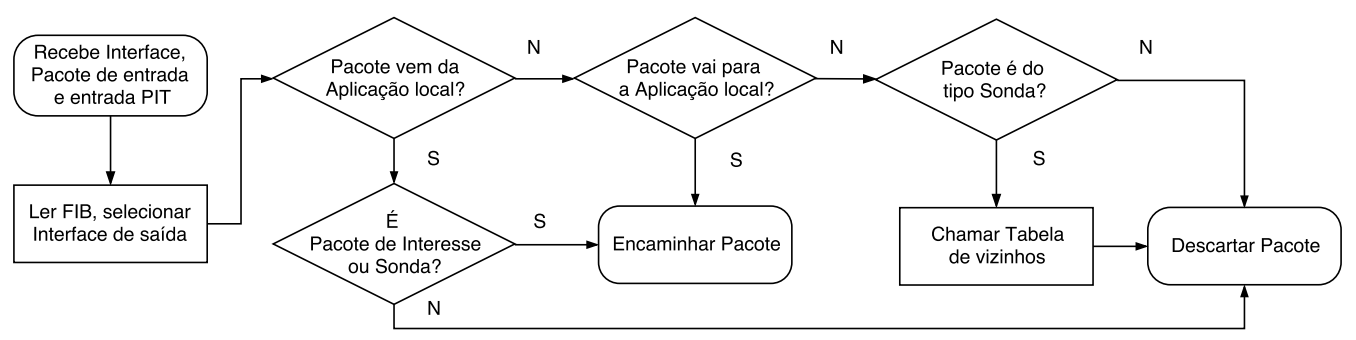

Figura 2. Estratégia de encaminhamento proposta. 


\subsection{Modificações na Camada de Estratégia da NDN}

A camada de estratégia da NDN é responsável por tomar as decisões quando o nó recebe um determinado pacote. Podem ser feitas consultas na CS, PIT e FIB se o pacote é do tipo interesse e consultas na PIT e CS se o mesmo é do tipo dados. Os pacotes de interesse são enviados para a estratégia de encaminhamento e os pacotes de dados são enviados para as camadas inferiores [Zhang et al. 2014]. Neste trabalho, as modificações realizadas na camada de estratégia, representam duas importantes decisões: (i) qual nó deve armazenar o dado, já que todos irão recebe-lo e, (ii) qual prioridade este dado terá sobre os já presentes na cache do nó selecionado na decisão (i), já que sua cache pode estar cheia. As respostas para estes dois questionamentos estão detalhadas nas subseções seguintes, 3.3.1 e 3.3.2, respectivamente.

\subsubsection{Escolha do nó para armazenar o dado}

Na NDN padrão os nós tendem a armazenar os conteúdos que passam por eles, através da política de colocação de dados LCE [Rehman and Kim 2017]. Contudo, em restrição à limitação de espaço na CS utilizam políticas de substituição de cache. Por outro lado, nas redes sem fio os pacotes trafegam via broadcast entre os nós. Portanto, em um ambiente de NDN sem fio um determinado dado é armazenado na cache de todos os nós presentes no domínio. Esses mecanismos apresentam-se ineficientes e desperdiçam os recursos computacionais dos dispositivos, através das replicações redundantes.

A fim de amenizar tais problemas, na solução proposta, sempre que um novo dado é gerado ou é requisitado a um determinado nó, este insere no campo idNodeBackup do pacote de dados, o identificador do nó que deve armazena-lo. Dessa forma, evita-se as replicações desnecessárias, para alcançar uma maior diversidade de conteúdos na rede. O processo de escolha do nó para armazenar a réplica do dado, bem como a inserção de sua prioridade, estão representados no Algoritmo 1. O algoritmo aplica a política de escalonamento Round-Robin na estrutura Tabel $a_{v i z i n h o s}$, com a finalidade de distribuir os dados entre os nós do domínio e evitar as replicações redundantes.

$\mathrm{Na}$ linha 2 é calculado o tempo estimado de permanência do nó na rede, linkTime, para atribuir uma prioridade aos seus dados. A prioridade máxima de um dado foi definida com o valor 100. Dados encaminhados por nós com baixo linkTime têm prioridade elevada (i.e., link $\mathrm{T}$ ime distante de cem e próximo de zero). Em seguida, na linha 3, é inserido no campo priorityData do pacote de dados a sua prioridade. Na linha 4, é verificada a estrutura Tabela $a_{v i z i n h o s}$ do nó, em busca de vizinhos conhecidos, a fim de selecionar o nó da WLAN que deve armazenar o conteúdo. Esse conteúdo pode ter sido recém gerado no produtor (e.g., vindo da aplicação), poderia já está presente na cache ou pode ser um reencaminhamento (e.g., vindo da rede), no caso do Ponto de Acesso (AP). Se o nó, ainda, não possuir vizinhos conhecidos, o dado é repassado para as camadas inferiores na pilha de protocolos NDN com o identificador do nó atual, linha 13.

Caso o nó já conheça algum vizinho é verificada a linha 5, onde é definido um limiar $\alpha$ que representa o raio da área $A^{c}$, em que $A$ corresponde a área virtual da cobertura do sinal do AP, como ilustrado na Figura 3. Tem-se, portanto, a área de cobertura real $B$ e uma subárea virtual $A$, com raio de menor alcance, $\log$ o $A \subset B$. O intervalo $A \cap B$ equivale a área segura onde os nós encontram-se relativamente próximos ao AP, por outro 


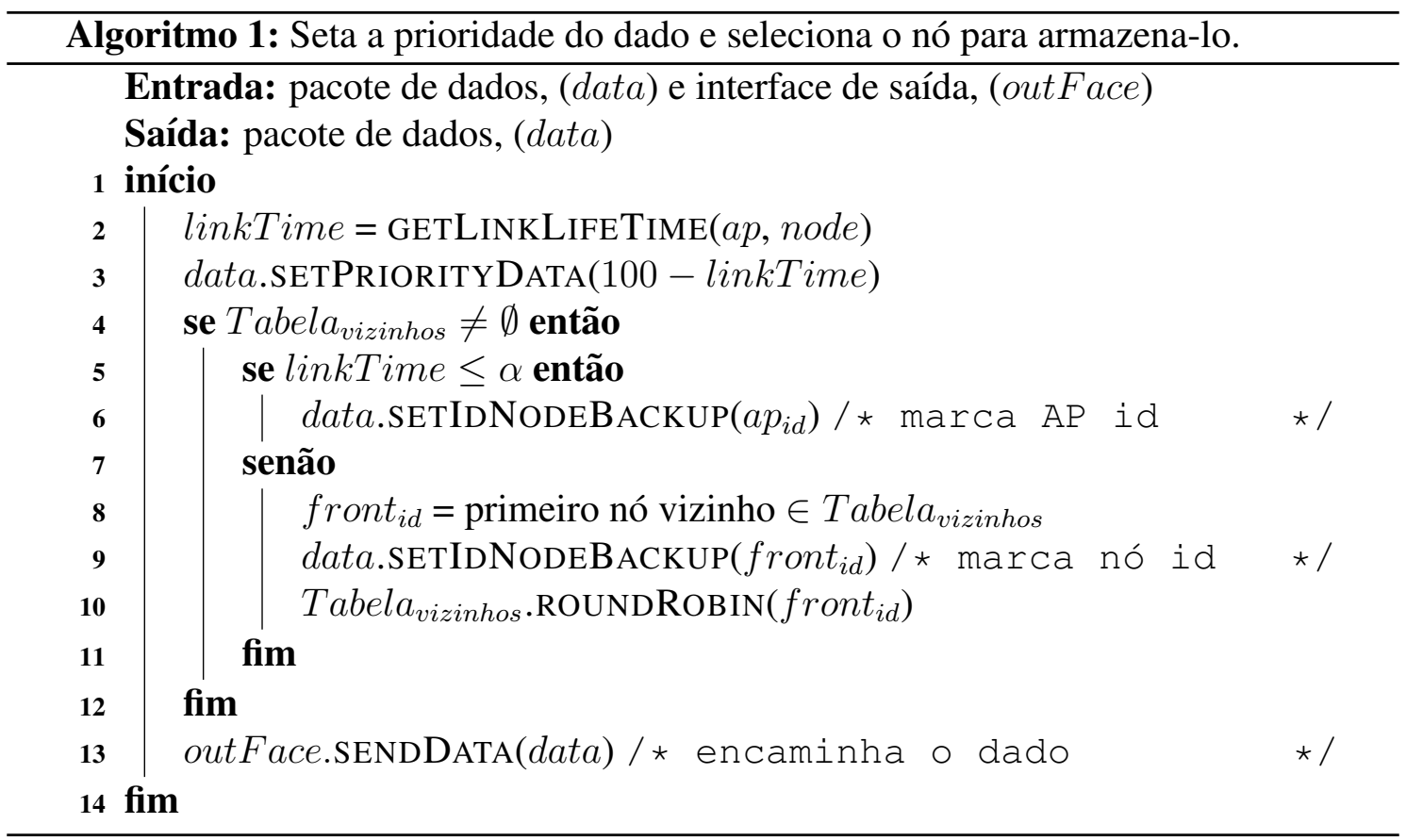

lado, o complemento $A^{c}$ compreende uma zona de alto índice de chances de ocorrer handoff. O tempo estimado de duração do nó na rede, linkTime, é comparado com $\alpha$, se o tempo de permanência do nó se encontra dentro da zona de handoff $\left(A^{c}\right)$, então o nó escolhe o AP para armazenar a cópia do dado e, para isso, simplesmente insere o identificador do AP no campo idNodeBackup do pacote de dados (linha 6).

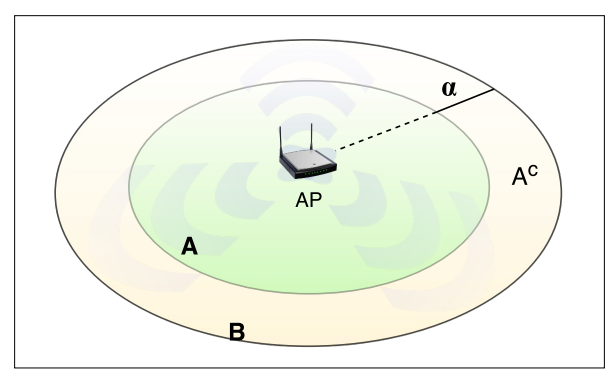

Figura 3. Alcance do sinal do AP representado pela área virtual A e área real B.

Caso o linkTime do nó seja maior que $\alpha$, então este encontra-se na área de cobertura $A$ (linha 7). Em seguida, da linha 8 a 11, realiza-se: a consulta na estrutura Tabela $a_{\text {vizinhos }}$ para obter o $i d$ do nó que irá armazenar o dado; a marcação do id no campo idNodeBackup do pacote de dados e; a execução do algoritmo de escalonamento Round-Robin na estrutura Tabela $a_{\text {vizinhos }}$, para agendar o nó que deve armazenar o próximo conteúdo a ser requisitado. Por fim, o dado é enviado, linha 13.

\subsubsection{Cálculo de prioridade do dado}

A prioridade do dado é utilizada pela política de substituição de cache (Subseção 3.4). O cálculo da prioridade do dado é invocado pelo Algoritmo 1 e é apresentado a seguir: 
sejam dois pontos $P$ e $Q$, com coordenadas vetoriais tridimensionais $x, y$ e $z, \log 0 P=$ $\left(p_{x}, p_{y}, p_{z}\right)$ e $Q=\left(q_{x}, q_{y}, q_{z}\right)$. Utilizando a fórmula da distância Euclidiana para obter o caminho mais curto, em forma de uma reta, entre os dois pontos, tem-se a Equação 1, que também pode fornecer a velocidade $v$ relativa dos nós.

$$
d_{P Q}=\sqrt{\left(p_{x}-q_{x}\right)^{2}+\left(p_{y}-q_{y}\right)^{2}+\left(p_{z}-q_{z}\right)^{2}}
$$

Aplicando a distância $d_{P Q}$, obtida na Equação 1, na variação da fórmula original de velocidade do Movimento Retilíneo Uniforme (MRU), temos a Equação 2, para o cálculo do tempo. Seja $r$, o raio de alcance máximo do sinal do AP, $r$ subtraído a distância atual do nó, $d_{P Q}$, corresponde a distância relativa para o limite da área de cobertura do AP. A predição do tempo $t$ é realizada, portanto, por meio da divisão da distância restante do nó pela sua velocidade.

$$
t=\frac{d_{P Q}}{v} \Rightarrow \frac{r-d_{P Q}}{v}
$$

O valor obtido de $t$ é retornado ao Algoritmo 1, na variável linkTime. Embora, considerou-se a velocidade do nó e a sua distância em relação ao AP, para calcular e atribuir uma prioridade aos seus dados, a prioridade poderia ser obtida de outras formas como, por exemplo, considerando a intensidade de sinal que o nó recebe do AP, o nível da carga de bateria disponível no nó ou a combinação de ambos.

\subsection{Política de Substituição de Cache}

Na NDN padrão existe algumas políticas de substituição de cache como, por exemplo, First-In First-Out (FIFO) e Last Recently Used (LRU) [Mastorakis et al. 2017]. Ambas têm características particulares, mas compartilham uma finalidade comum de substituir dados na cache dos nós. Sempre que um dado é transmitido na rede sem fio, os demais nós o receberão via broadcast e irão armazena-lo. Isso faz com que uma mesma cache seja espelhada em todas as outras da rede, tornando os recursos desperdiçados, por armazenar dados que já encontram-se armazenados em outros nós.

A solução proposta apresenta uma nova política de substituição de cache com base na prioridade do dado. A política, no entanto, precisa atuar em conjunto com a camada de estratégia modificada, já apresentada nas seções anteriores, onde é feita a marcação no pacote de dados informando a sua prioridade e o identificador do nó que deve armazenalo. A política de substituição de cache desenvolvida está representada no Algoritmo 2. Todos os nós da WLAN, inclusive o AP, devem possuir essa política instalada em sua pilha de protocolos NDN, para alcançar o melhor desempenho da solução proposta.

$\mathrm{Na}$ linha 1 é definida a função que gerencia a cache em cada nó da WLAN. Essa função recebe pacote de dados como argumento e é acionada imediatamente após um pacote desse tipo ser armazenado na CS do nó. Na linha 2 é verificado se a capacidade da cache já atingiu seu limite de armazenamento de dados. Caso negativo, então o dado permanece na CS e o algoritmo se encerra (linhas 14 e 15).

Caso a CS tenha atingido seu limite, então é verificado se o dado em questão é destinado ao nó atual (i.e., o idNodeBackup do dado é igual ao id do nó), linha 3. Se 


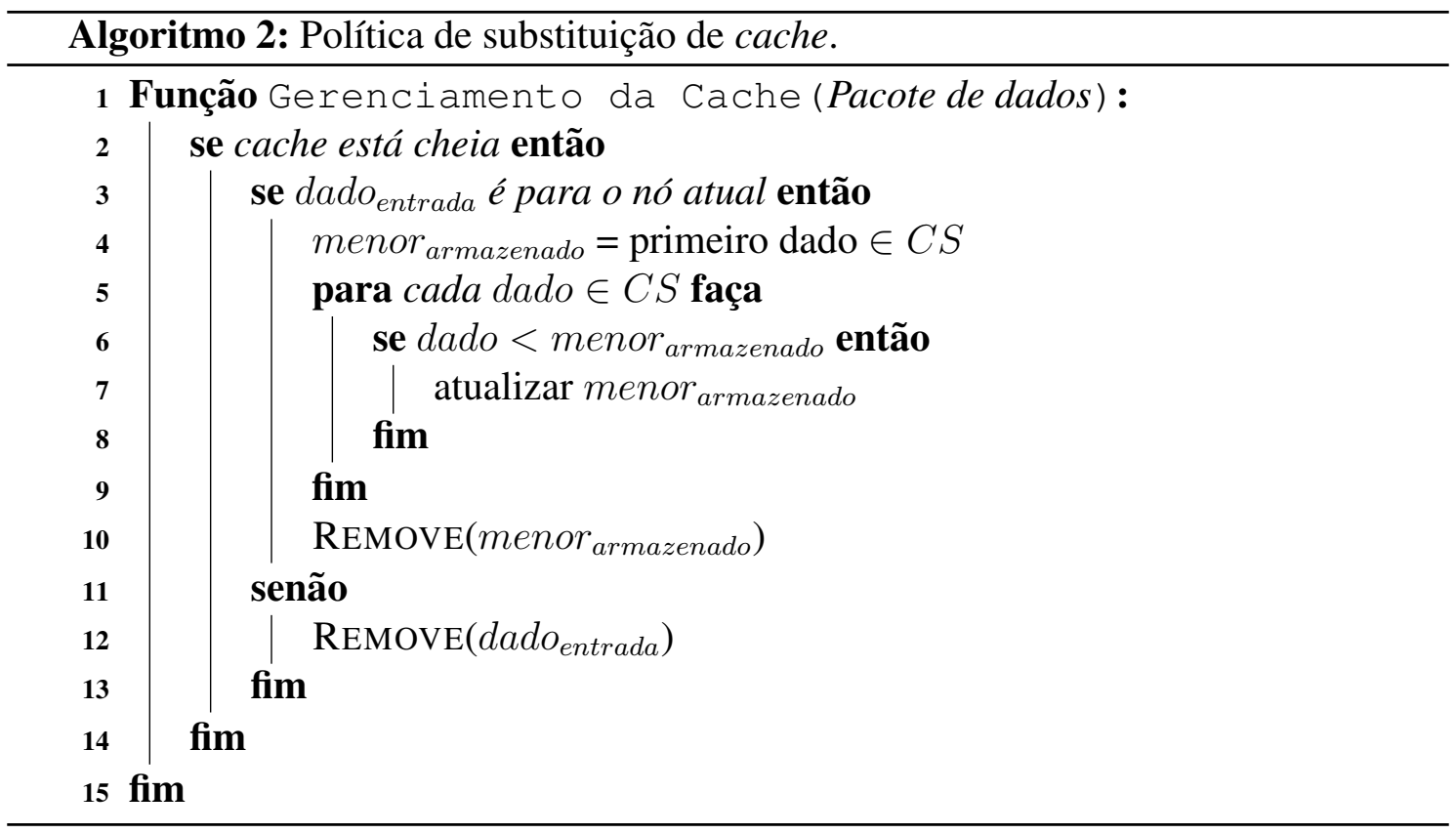

o dado não é para o nó atual (linha 11), o algoritmo apenas o descarta (linha 12). Por outro lado, se o dado é para o nó atual, este busca em sua cache o dado com menor prioridade dentre os armazenados, linhas 4 a 9. Depois de encontrar o dado de menor prioridade, este é removido da cache do nó (linha 10). Após esse processo, apenas os dados de maior prioridade permanecerão na cache, obedecendo a capacidade da mesma.

\section{Estudo Experimental e Análise dos Resultados}

Esta seção apresenta o cenário de avaliação da proposta, o ambiente de experimentação, a metodologia utilizada e a análise dos resultados obtidos.

\subsection{Cenário de Avaliação}

O cenário de avaliação da solução proposta está representado na Figura 4. Nota-se que há quatro consumidores no domínio do AP1, apesar dos consumidores estarem em uma WLAN, não é considerado o handoff dos mesmos. Dessa forma, apenas os produtores no domínio do AP2 efetuam handoff em direção ao AP3. O AP2, por sua vez, pode possuir até 30 nós móveis, em que até 9 produtores podem efetuar handoff simultaneamente. Além disso, o AP2 possui um produtor que, embora móvel, não ultrapassa sua área de cobertura de sinal. A razão disso, é tornar o ambiente mais realístico em que os dados desse produtor competem com os dados dos demais produtores por espaço na cache dos nós. Vale ressaltar, ainda, que a cache dos consumidores, AP1 e roteadores foi desabilitada para enfatizar a real diferença entre a solução proposta e a NDN padrão.

Os consumidores requisitam dados, a cada produtor, seguindo uma taxa randômica (frequência) que varia de 5 a 8 interesses por segundo, no entanto, o intervalo entre os interesses segue uma distribuição exponencial (i.e., com média $=1$ / frequência). O consumidor C4 é um caso especial que solicita dados tanto ao produtor destinado exclusivamente a competir por espaço em cache, quanto aos demais produtores. Nas experimentações, as requisições de dados se iniciaram com distinção de 10 segundos entre os consumidores, 


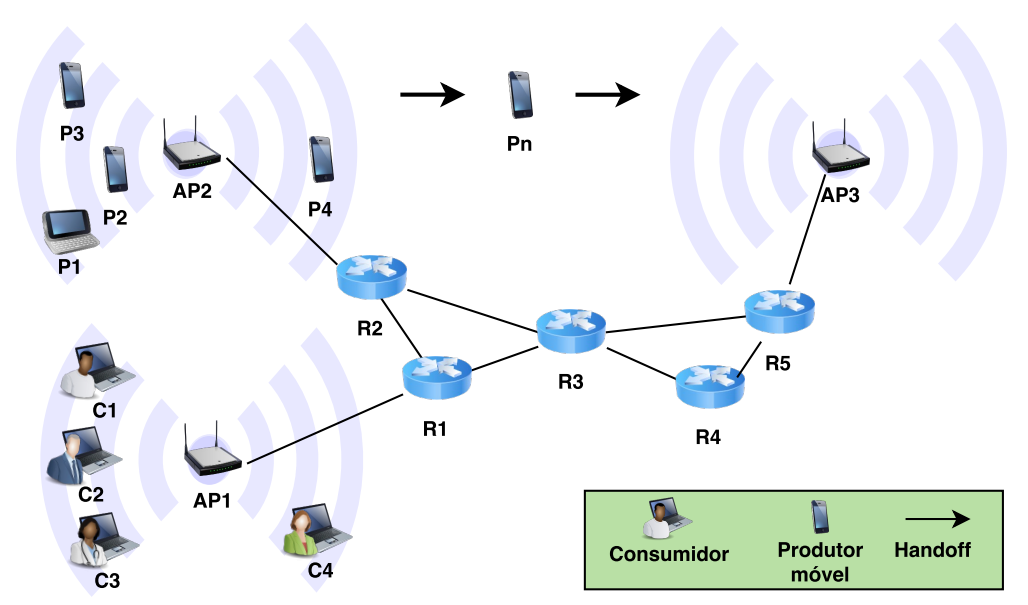

Figura 4. Cenário de avaliação da proposta.

ou seja, $\mathrm{C} 1=0 \mathrm{~s}, \mathrm{C} 2=10 \mathrm{~s}, \mathrm{C} 3=20 \mathrm{~s}$ e $\mathrm{C} 4=30 \mathrm{~s}(\mathrm{C} 4$ inicia requisições, também, no tempo 0 segundo para o produtor que não realiza handoff). É importante destacar que as simulações foram longas o suficiente para que os produtores móveis partissem do domínio do AP2 e chegassem ao AP3. O período transiente é tratado, portanto, através da característica acima mencionada da simulação.

\subsection{Ambiente de Experimentação}

Para avaliar o desempenho da solução proposta, foi desenvolvido o ambiente de experimentação no simulador de redes, baseado em eventos discretos, ndnSIM, no qual é um módulo do NS-3 ${ }^{1}$ específico para NDN. Os parâmetros empregados nos experimentos, comuns a ambas as soluções (i.e., NDN padrão e solução proposta), estão presentes na Tabela 1. Além destes, a NDN padrão assumiu a estratégia de encaminhamento de interesses best-route e a política de substituição de cache LRU, enquanto a solução proposta assumiu a estratégia e política desenvolvidas, respectivamente (subseções 3.2 e 3.4). Os parâmetros ausentes na Tabela 1 assumiram valores padrão do ndnSIM.

Tabela 1. Parâmetros da Simulação.

\begin{tabular}{ll}
\hline Parâmetro & Valor \\
\hline Simulador de redes & ndnSIM \\
Modelo de rádio propagação & Nakagami \\
Modelo de mobilidade dos nós em handoff & ConstantVelocity \\
Modelo de mobilidade dos nós na WLAN & RandomWaypoint \\
Alcance de transmissão do AP & 100 metros \\
Protocolo de enlace & $802.11 \mathrm{a}$ \\
Tamanho do pacote de dados & $1 \mathrm{~KB}$ \\
\hline
\end{tabular}

\subsection{Metodologia de Avaliação}

A avaliação foi dividida em duas etapas: (i) um modelo de planejamento de experimentos fatorial completo $2^{k}$, recomendado por [Jain 1991]. Em seguida, na etapa (ii), uma análise de desempenho com os fatores mais significativos obtidos na primeira etapa.

\footnotetext{
${ }^{1}$ https://www.nsnam.org/
} 
Na etapa (i) aferiu-se os impactos que cada fator exerce sobre a variável de resposta taxa de satisfação de interesse. Esta foi escolhida pois representa o sucesso do recebimento de dados por parte dos consumidores. Os valores assumidos por $k$ (i.e., $2^{k}$ ) foram os cinco fatores listados na Tabela 2. Os fatores e níveis foram definidos com referência aos presentes na literatura e de modo a abranger a maior quantidade de casos suscetíveis de ocorrências em um ambiente real. Nesta etapa, do projeto fatorial, apenas os valores mínimo e máximo são considerados por fator e cada experimento foi replicado 5 vezes para obter a média com $95 \%$ de confiança.

Tabela 2. Fatores e níveis do Projeto Fatorial.

\begin{tabular}{llll}
\hline ID & Fator & mín. & máx. \\
\hline A & Velocidade dos produtores em handoff & $1 \mathrm{~m} / \mathrm{s}$ & $3 \mathrm{~m} / \mathrm{s}$ \\
B & Quantidade de produtores em handoff & $1 \mathrm{nó}$ & $9 \mathrm{nós}$ \\
C & Velocidade dos nós & $0 \mathrm{~m} / \mathrm{s}$ & $2 \mathrm{~m} / \mathrm{s}$ \\
D & Tamanho da cache dos nós & 80 pacotes & 160 pacotes \\
E & Quantidade de nós na WLAN & 10 nós & 30 nós \\
\hline
\end{tabular}

O resultado da influência dos fatores sobre a variável de resposta, está representado através do gráfico pareto na Figura 5. É possível observar que os fatores dominantes, acima da linha vermelha, são: B (Quantidade de produtores em handoff) e A (Velocidade dos produtores em handoff), juntos corresponderam a pouco mais de $80 \%$ do impacto na variável de resposta seguindo, portanto, o princípio de pareto. O fator A está intrinsecamente relacionado à predição e detecção do produtor em handoff. Os demais fatores exerceram limitada significância para o sistema em relação aos fatores A e B.

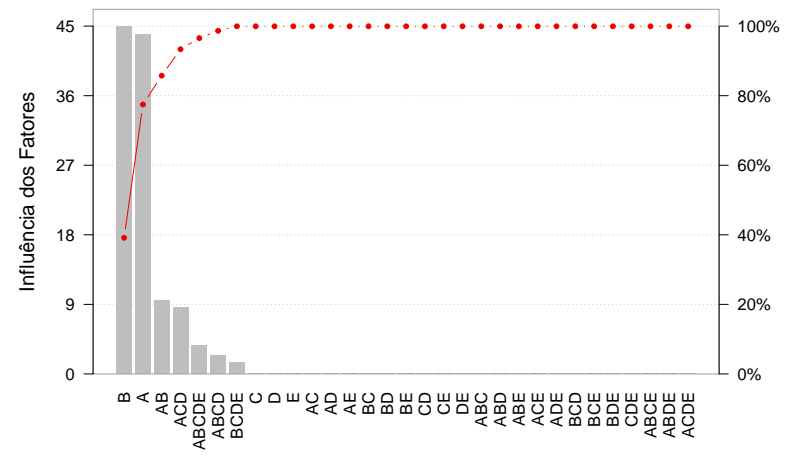

Figura 5. Projeto Fatorial.

Na etapa (ii) após obter os fatores mais influentes para o sistema, de acordo com a etapa (i), considerou-se A e B para uma análise mais profunda da proposta. Para tanto, foi incrementado mais um nível aos fatores obtidos, dessa forma $A=\{1,2,3\} \mathrm{m} / \mathrm{s}$ e $B=\{1$, $5,9\}$ nós, os demais fatores ( $C$, D e E) da Tabela 2, nesta etapa assumiram os seus valores máximos sem qualquer variação. Nesta etapa, cada experimento foi replicado 10 vezes com diferentes sementes para obter a média com nível de confiança de 95\%, seguindo a distribuição $t$-student. Além disso, as seguintes métricas foram consideradas na análise de desempenho da proposta, do ponto de vista dos consumidores e da WLAN: 
- Taxa de satisfação de interesses - consiste na razão entre os pacotes de dados recebidos pelos pacotes de interesses enviados. Representa o quão o sistema é eficaz na entrega de dados aos consumidores.

- Tempo de reposta - se dá pela diferença de tempo entre o recebimento do pacote de dados pelo envio do primeiro pacote de interesse para o respectivo dado, ou seja, inclui os tempos de retransmissões. Representa o quão o sistema é eficiente na entrega de dados aos consumidores.

- Pacotes encaminhados no domínio da WLAN - consiste na média total da quantidade de pacotes de interesses, dados, nacks ${ }^{2}$ e sondas. O tráfego desses pacotes reflete o estado da rede, isso é importante para inferir as proporções que os mesmos são encaminhados e a consequência que isso pode ocasionar à rede.

\subsection{Análise dos Resultados}

A média geral da taxa de satisfação de interesses que cada consumidor obteve, em ambas as soluções, está representada na Figura 6. Nota-se que para todos os casos (i.e., todas as interações dos níveis dos fatores) a solução proposta supera a NDN padrão e isso se reflete em todos os consumidores. Dessa forma, a solução proposta alcança um nível superior na entrega de dados e, consequentemente, na taxa de satisfação de interesses.

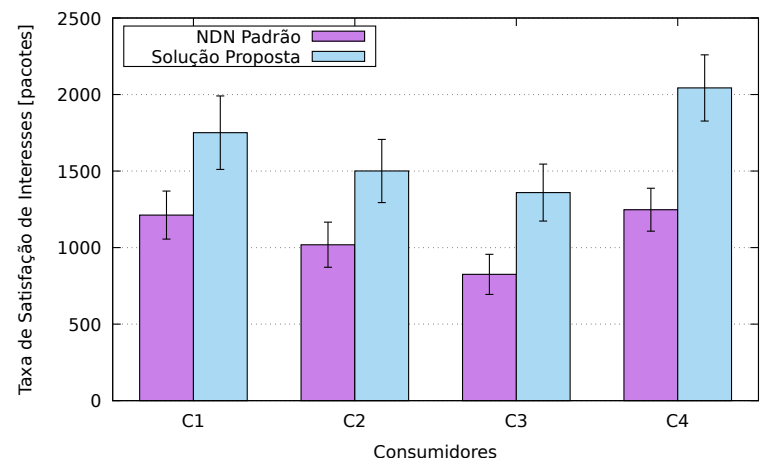

Figura 6. Taxa de satisfação de interesses por consumidor.

A média geral do atraso, para obter dados, de todos os pacotes de interesses encaminhados pelos consumidores, incluindo retransmissões, está ilustrada na Figura 7. É possível observar que a solução proposta, novamente, apresenta um desempenho superior à NDN padrão, de modo a obter atrasos inferiores em cada consumidor.

A Figura 8 representa o tráfego de pacotes na WLAN, nos cenários com a NDN padrão e a solução proposta. A Figura 8 (a) mostra os pacotes de interesse encaminhados, nota-se que as duas soluções encontram-se estatisticamente iguais. A Figura 8 (b), ilustra a quantidade de pacotes de dados trafegados na WLAN, em que a solução proposta supera a NDN padrão, isso se dá pelo fato de que mais dados são encontrados nas cache dos nós. Além disso, a solução proposta não encaminha pacotes nacks o que torna a rede menos congestionada, conforme ilustra a Figura 8 (c). A NDN padrão congestiona a rede com pacotes nacks, principalmente, quando os produtores fazem handoff e os interesses que chegam não conseguem ser atendidos. A solução proposta bloqueia os nacks, por meio da estratégia de encaminhamento criada, no entanto, insere pacotes do tipo sonda. A Figura

\footnotetext{
${ }^{2}$ Informam quando o destino encontra-se inalcançável [Zhang et al. 2014].
} 


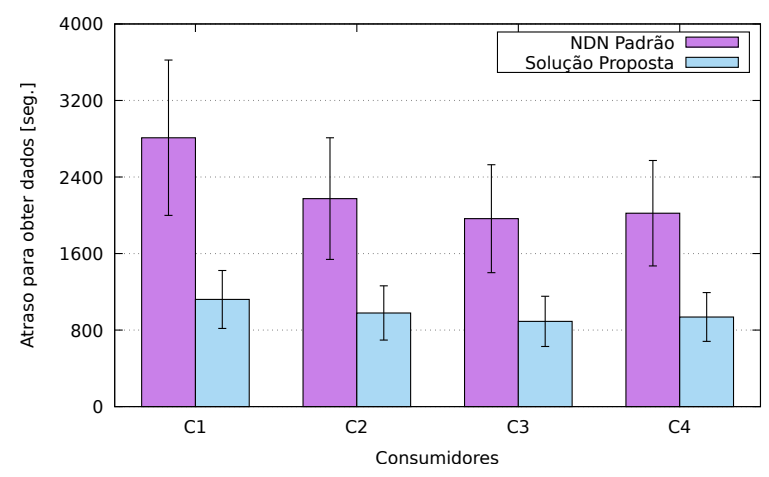

Figura 7. Atraso para obter dados por consumidor.

8 (d), destaca a quantidade de sondas inseridas, observa-se que esses pacotes representam uma baixa carga para a rede e, por isso, não afetam o desempenho da mesma.

(a)

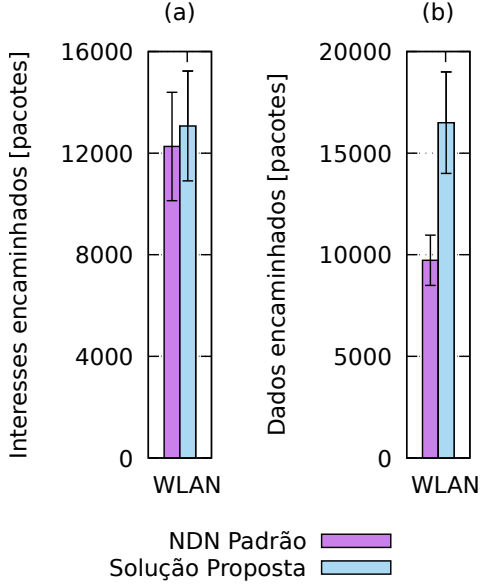

(c)

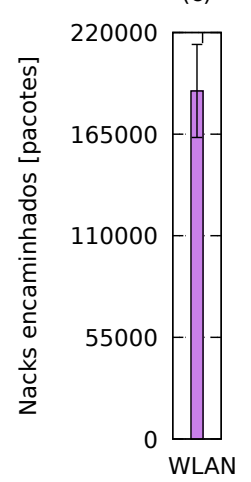

(d)

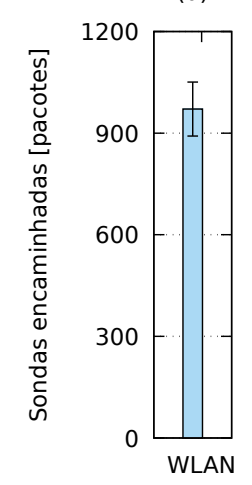

Figura 8. Tráfego de pacotes encaminhados na WLAN.

\section{Conclusão e Trabalhos Futuros}

A NDN se apresenta como uma arquitetura promissora para a Internet do Futuro. No entanto, apesar de suportar a mobilidade dos consumidores a mobilidade dos produtores não tem suporte adequado, principalmente, quando estes perdem a conexão com a rede. Uma vez que em NDN os dados podem ser mantidos na cache dos nós, este trabalho se concentrou em desenvolver uma solução para amenizar os problemas causados à rede pela mobilidade dos produtores, de modo a priorizar os dados dos produtores mais propensos a efetuarem handoff nos demais nós da rede sem fio. Dessa forma, aumenta-se a diversidade de conteúdo na rede e, consequentemente, as chances de resposta aos interesses que chegam no período de indisponibilidade do produtor.

Os resultados obtidos das simulações mostraram que a solução proposta mantém a taxa de satisfação de interesses elevada mesmo com a indisponibilidade do produtor e com uma redução significativa do tempo de resposta para obter os dados, comparada à NDN padrão. Além disso, a solução proposta segue o princípio da NDN e é genérica, podendo ser aplicada a outros cenários de redes sem fio (e.g., 5G, Mesh, Ad Hoc). Para 
trabalhos futuros pretendemos avaliar a solução proposta nos cenários citados e com a mobilidade simultânea de produtores e consumidores.

\section{Agradecimentos}

Os autores agradecem o apoio da FAPESB e do CNPq.

\section{Referências}

Cisco (2017). Cisco Visual Networking Index: Global Mobile Data Traffic Forecast Update, 2016-2021. Technical report.

Farahat, H. and Hassanein, H. (2016). Optimal caching for producer mobility support in Named Data Networks. In 2016 IEEE International Conference on Communications (ICC), pages 1-6. IEEE.

Ge, J., Wang, S., Wu, Y., Tang, H., and E, Y. (2016). Performance improvement for source mobility in named data networking based on global-local FIB updates. Peer-to-Peer Networking and Applications, 9(4):670-680.

Hahm, O., Baccelli, E., Schmidt, T. C., Wählisch, M., Adjih, C., and Massoulié, L. (2017). Low-power Internet of Things with NDN \& Cooperative Caching. In Proceedings of the 4th ACM Conference on Information-Centric Networking, ICN '17, pages 98-108, New York, NY, USA. ACM.

Huynh, T., Priyono, O., Lee, S., and Hwang, W. (2017). Simultaneous mobility of data sources and content requesters in content-centric networking. Peer-to-Peer Networking and Applications, 10(1):31-44.

Jain, R. (1991). The art of computer systems performance analysis: techniques for experimental design, measurement, simulation, and modeling. John Wiley \& Sons.

Lehmann, M. B., Barcellos, M. P., and Mauthe, A. (2016). Providing producer mobility support in NDN through proactive data replication. In NOMS 2016 - 2016 IEEE/IFIP Network Operations and Management Symposium, pages 383-391. IEEE.

Mastorakis, S., Afanasyev, A., and Zhang, L. (2017). On the evolution of ndnSIM: an open-source simulator for NDN experimentation. ACM Computer Communication Review.

Panwar, G., Tourani, R., Mick, T., Mtibaa, A., and Misra, S. (2017). DICE: Dynamic Multi-RAT Selection in the ICN-enabled Wireless Edge. In Proceedings of the Workshop on Mobility in the Evolving Internet Architecture, MobiArch '17, pages 3136, New York, NY, USA. ACM.

Rehman, R. A. and Kim, B. (2017). LOMCF: Forwarding and Caching in Named Data Networking Based MANETs. IEEE Transactions on Vehicular Technology, 66(10):9350-9364.

Zhang, L., Afanasyev, A., Burke, J., Jacobson, V., Claffy, K., Crowley, P., Papadopoulos, C., Wang, L., and Zhang, B. (2014). Named data networking. ACM SIGCOMM Computer Communication Review, 44(3):66-73.

Zhang, Y., Afanasyev, A., Burke, J., and Zhang, L. (2016). A survey of mobility support in Named Data Networking. In 2016 IEEE Conference on Computer Communications Workshops (INFOCOM WKSHPS), pages 83-88. IEEE. 